\title{
Metabolic responses to high-fat diets rich in MUFA v. PUFA
}

\author{
Kristine R. Polley ${ }^{1}$, Mary K. Miller ${ }^{2}$, Mollie Johnson ${ }^{2}$, Roger Vaughan ${ }^{2}$, Chad M. Paton ${ }^{1,3}$ and \\ Jamie A. Cooper ${ }^{1,2 *}$ \\ ${ }^{1}$ Department of Foods and Nutrition, University of Georgia, 305 Sanford Drive, Athens, GA 30602, USA \\ ${ }^{2}$ Department of Nutritional Sciences, Texas Tech University, 1301 Akron Street, Lubbock, TX 79409-1270, USA \\ ${ }^{3}$ Department of Food Science and Technology, University of Georgia, 100 Cedar Street, Athens, GA 30602, USA
}

(Submitted 20 November 2017 - Final revision received 26 February 2018 - Accepted 15 April 2018)

\section{Abstract}

Dietary fatty acid (FA) composition may influence metabolism, possibly affecting weight management. The purpose of this study was to compare the effects of a 5-d diet rich in PUFA $v$. MUFA. A total of fifteen normal-weight men participated in a randomised cross-over design with two feeding trials ( $3 \mathrm{~d}$ lead-in diet, pre-diet visit, 5-d PUFA- or MUFA-rich diet, post-diet visit). The 5-d diets (50\% fat) were rich in either PUFA ( $25 \%$ of energy) or MUFA ( $25 \%$ of energy). At pre- and post-diet visits, subjects consumed breakfast and lunch test meals, rich in the FA for that 5-d diet. Indirect calorimetry was used for $4 \mathrm{~h}$ after each meal. There were no treatment differences in fasting metabolism acutely or after the 5-d diet. For acute meal responses before diet, RER was higher for PUFA $v$. MUFA (0.86 (SEM 0.01) $v \cdot 0 \cdot 84$ (SEM 0.01), $P<0 \cdot 05$ ), whereas diet-induced thermogenesis (DIT) was lower for PUFA $v$. MUFA (18.91 (sEm 1.46) $v$. 21.46 (SEm 1.34) kJ, $P<0.05$ ). After the 5 -d diets, the change in RER was different for PUFA $v$. MUFA $(-0.02$ (SEM 0.01) $v .0 .00$ (SEM 0.01 ), $P<0 \cdot 05$ ). Similarly, the change in fat oxidation was greater for PUFA $v$. MUFA (0.18 (SEM 0.07) $v$. 0.04 (SEM 0.06) g, $P<0.05$ ). In conclusion, acutely, a MUFA-rich meal results in lower RER and greater DIT. However, after a 5-d high-fat diet, the change in metabolic responses was greater in the PUFA diet, showing the metabolic adaptability of a PUFA-rich diet.

Key words: Dietary fatty acids: PUFA: MUFA: RER: Metabolism

Dietary fats are implicated in the development of several metabolic diseases including obesity, diabetes and $\mathrm{CVD}^{(1)}$. Over-consumption of energy-dense foods, which is typically associated with a high fat (HF) content in foods, is a main contributor to positive energy balance ${ }^{(2)}$. In recent years, research has suggested that the dietary fatty acid (FA) composition of a HF diet may act differentially on energy usage and storage, affecting weight gain and loss ${ }^{(3,4)}$.

Decreased fat oxidation has been associated with an increase in body fat ${ }^{(5)}$. Therefore, it may be important to identify foods and diets that increase fat oxidation for weight management. Early animal and human studies have consistently revealed greater oxidation of unsaturated FA compared with $\mathrm{SFA}^{(6-8)}$, possibly contributing to recommendations centred around increasing unsaturated fat consumption and limiting SFA intake $^{(1)}$. Differences between rates of fat oxidation among various unsaturated FA also exist. Early human studies using isotope labelling techniques indicate differences in oxidation between MUFA and PUFA ${ }^{(9,10)}$. Jones et al. ${ }^{(9)}$ found that MUFA were more highly oxidised than $n-6$ PUFA in normal-weight men. Delany et al. ${ }^{(10)}$ found that again MUFA were more highly oxidised than $n$-6 PUFA; however, $n-3$ PUFA were the most highly oxidised FA compared with both MUFA and n-6 PUFA. More recent research using indirect calorimetry to measure total fat oxidation rates after acute meal challenges have found no differences between these unsaturated fats ${ }^{(11-13)}$.

Diet-induced thermogenesis (DIT) is important in the regulation of energy balance ${ }^{(5)}$, and, similar to fat oxidation, reduced activity of thermogenesis has been associated with obesity ${ }^{(14,15)}$. Studies to date have consistently reported greater DIT after consumption of unsaturated fats compared with saturated fats ${ }^{(8,12,13,16)}$. Only three studies to date have compared acute meal challenges rich in MUFA $v$. PUFA with mixed findings ${ }^{(11-13)}$. Studies have yet to observe the longer-term effects of consuming a diet rich in MUFA $v$. PUFA on DIT or energy expenditure (EE).

It is important to note that the studies mentioned thus far have all been acute or single-meal challenge studies. This may be limiting because postprandial oxidation rates following an acute meal may differ based on historical dietary fat consumption and prior body fat composition. A longer-term diet intervention that can influence metabolic changes or adaptations may more accurately define the differential effects of altering dietary FA

Abbreviations: CSO, cottonseed oil; DIT, diet-induced thermogenesis; EE, energy expenditure; FA, fatty acid; HF, high fat; OO, olive oil.

* Corresponding author: J. A. Cooper, fax +1 706542 5059, email jamie.cooper@uga.edu 
composition $^{(3)}$. Some longer-term diet studies comparing HF diets rich in MUFA $v$. SFA ${ }^{(17-19)}$ and PUFA $v$. SFA ${ }^{(6,20,21)}$ have been conducted with significant metabolic differences. However, research exploring the effects of longer-term HF diets rich in PUFA $v$. MUFA on metabolism is lacking. In our laboratory, we have recently shown that a diet high in PUFA resulted in greater fat oxidation after a SFA-rich meal compared with a control diet high in MUFA ${ }^{(22)}$

Because of the inconsistent results of previous studies and the lack of research on longer-term ingestion of different types of unsaturated FA-rich diets, the purpose of this study was to compare the effect of a HF diet rich in MUFA $v$. PUFA on metabolism in normal-weight men. The primary outcome was to determine the effect of a 5-d diet intervention on fasting and postprandial substrate oxidation and DIT in our PUFA-rich $v$. MUFA-rich diets. On the basis of studies previously performed in our laboratory ${ }^{(13,22)}$, we hypothesised that the HF, PUFA-rich diet would lead to greater fasting and postprandial fat oxidation and DIT compared with the HF, MUFA-rich diet. Our secondary outcome was to evaluate the acute meal response, before the diet intervention, of consuming a HF meal rich in MUFA $v$. PUFA on metabolism. On the basis of previous findings, we hypothesised that there would be no differences in total fat oxidation and DIT following acute consumption of the PUFA-rich $v$. MUFA-rich HF meals.

\section{Methods}

\section{Study design}

This study was a single-blind, randomised cross-over design consisting of two different feeding trials. The study protocol included a baseline visit (RMR measured), followed by two outpatient feeding trials, and is outlined in Fig. 1. The feeding trials consisted of a 3 -d lead-in diet, a pre-diet testing visit (visit 1), a 5-d feeding protocol (rich in either MUFA or PUFA) and a post-diet testing visit (visit 2). Before all visits, participants fasted for 8-12 h and avoided any vigorous exercise for $12 \mathrm{~h}$. After the first trial, there was a 2- to 4-week washout period. Participants then completed trial 2. The only difference between the trials was the type of unsaturated fat provided in the HF diet during the 5-d feeding period. The HF diets were rich in either MUFA or PUFA. Participants were randomised to either receive the HF, PUFA-rich diet first or the HF, MUFA-rich diet first following their baseline visit using Research Randomizer (www.randomizor.org). This study was single-blinded, so the participants were not aware of which diet they were receiving first or second.

\section{Participants}

A total of fifteen healthy, normal-weight, sedentary adult men were recruited for the study. Inclusion criteria were men between the ages of 18 and 45 years, performing $<3 \mathrm{~h}$ of structured exercise per week and normal-weight status based on a BMI between 18 and $24.9 \mathrm{~kg} / \mathrm{m}^{2}$ or body composition analysis using dual X-ray absorptiometry (DXA ${ }^{\mathrm{TM}}$, Discovery A; Hologic Inc.), with a body fat percentage less than $24 \%$ to qualify. The exclusion criteria included the following: weight loss or gain exceeding $5 \%$ of body weight in the past 3 months; regular exercise $>3 \mathrm{~h} /$ week; plans to lose weight or begin a weight-loss programme between initiation of the study and final testing; anyone who was vegan or vegetarian; medication use; chronic or metabolic disease, gastrointestinal disorder or history of medical or surgical events that could affect digestion or swallowing; any supplement use other than a daily multivitamin; blood or plasma donation within $20 \mathrm{~d}$ before initiation of study; or tobacco use. Subjects were recruited through flyers, campus emails and word of mouth. This study was conducted according to the guidelines laid down in the Declaration of Helsinki, and all procedures involving human subjects were approved by the Western Institutional Review Board and written informed consent was obtained before beginning study procedures.

\section{Procedures}

Baseline visit. Participants reported to the Human Nutrition Laboratory after an overnight fast (no food or drink except water for 8-12 h). Anthropometric measurements (height,

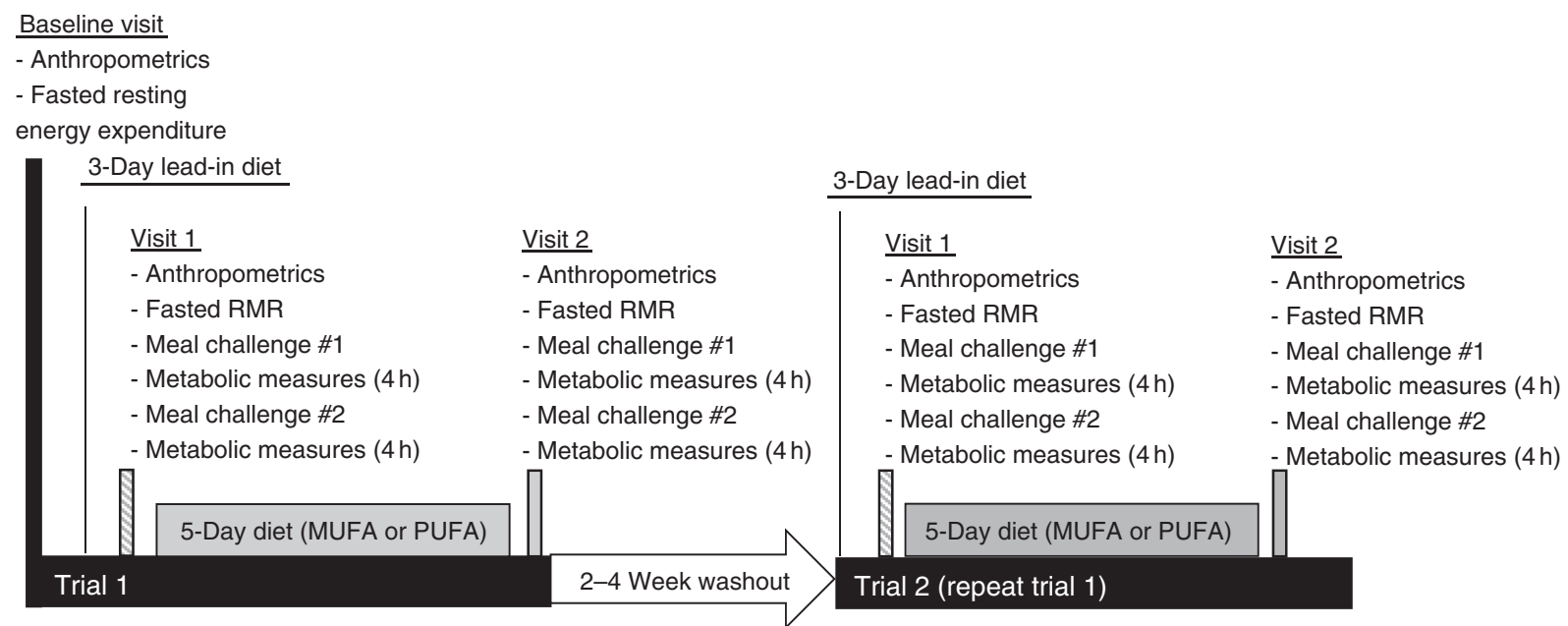

Fig. 1. Time course of study visits and measurements taken at each visit. 
weight and body composition) were taken, followed by RMR measurement with the ParvoMedics TrueOne ${ }^{\circledR} 2400$ Canopy System (Parvomedics) for 30 min using standardised conditions. Briefly, subjects were asked to stay awake and motionless in a supine position while a plastic hood was placed over their head to measure oxygen consumption and carbon dioxide production. Respiratory gases were used to calculate RMR using the Weir equation ${ }^{(23)}$. Participants' estimated total daily energy needs for the 3-d lead-in diet, 5-d diet and meal challenges at visits 1 and 2 were based on their calculated RMR multiplied by an average US physical activity value of $1.65^{(24)}$.

Lead-in diet. For $3 \mathrm{~d}$ before visit 1, participants were provided with a lead-in diet that was representative of the standard American diet (50\% carbohydrate, 35\% fat and 15\% protein, Table 1). Participants were instructed to consume all foods provided, and no additional foods or beverages besides water were allowed. Total energy content was based on the participant's estimated energy needs calculated from the RMR measurement at the 'Baseline visit' section described above.

Pre-diet testing visit (visit 1). Following the 3-d lead-in diet, participants arrived at the laboratory the next morning at 06.45 hours in a fasted state (8-12 h fast) and $12 \mathrm{~h}$ without exercise. Height, weight, blood pressure, waist and hip circumference and body fat percentage using DXA were measured. Indirect calorimetry was used to measure RMR and fasting substrate oxidation (RER and fat and carbohydrate oxidation) for $30 \mathrm{~min}$, as described above. Following indirect calorimetry measurement, participants ingested a HF liquid meal rich in either MUFA or PUFA (Table 2). The nutrient content of the meal was designed to provide $35 \%$ of the participant's estimated total daily energy needs (determined from RMR at 'Baseline visit' section). The liquid meals contained 1\% milk, whey chocolate protein, chocolate syrup and added oil. The oil, either olive oil (OO) or cottonseed oil (CSO), depended on the treatment condition (OO for MUFA or CSO for PUFA). We analysed the FA composition of each of the oils used in this study. The OO consisted of $19 \cdot 3 \%$ SFA, $67 \cdot 1 \%$ MUFA and $13.6 \%$ PUFA. The CSO consisted of $22.7 \%$ SFA, $19.9 \%$ MUFA and $57.4 \%$ PUFA. Participants consumed this liquid meal at breakfast (08.00 hours) and another identical meal again $4 \mathrm{~h}$ later for lunch (12.00 hours).

Table 1. Nutrient content for each high-fat (HF) diet

\begin{tabular}{lccc}
\hline Composition & Lead-in diet & PUFA-rich HF diet & MUFA-rich HF diet \\
\hline Percentage of total energy from & & \\
Protein & 15.0 & 15.0 & 15.0 \\
Carbohydrate & 50.0 & 35.0 & 35.0 \\
Fat & 35.0 & 50.0 & 50.0 \\
Percentage of energy from fatty & acid of interest & \\
MUFA & 16.0 & 9.7 & 31.5 \\
PUFA & 7.6 & 26.7 & 6.6 \\
$n-6$ PUFA & 7.6 & 26.3 & 5.7 \\
$n-3$ PUFA & 0.0 & 0.4 & 0.9 \\
SFA & 11.4 & 13.4 & 10.4 \\
\hline
\end{tabular}

After breakfast meal ingestion, respiratory gases were once again measured to determine EE and fuel utilisation. Measurements were obtained for a total of $8 \mathrm{~h}$ postprandially ( $4 \mathrm{~h}$ post breakfast and $4 \mathrm{~h}$ post lunch). Specifically, data were collected for a 20-min period followed by a 10-min break. During the 20 min of data collection, participants remained awake but were instructed not to move. During the 10-min break, participants remained seated with minimal movement. Participants were also given $4 \mathrm{oz}$ of water in each hour.

Dietary intervention. Following visit 1, participants began the 5-d, HF feeding trial rich in either MUFA or PUFA (Table 1). The order of the feeding trials was randomised. On days 1-5, participants reported to the laboratory between 07.00 and 10.00 hours to receive their breakfast, which was given as a shake. After the meal, participants were then given the rest of their food and beverages for the day and instructed to consume all the food provided and abstain from any other food or drink aside from water. All food items were weighed and prepared by research personnel. The 5 -d diet consisted of $50 \%$ of total energy from fat, $35 \%$ from carbohydrates and $15 \%$ from protein. For the HF portion, diets were prepared using either CSO (for the PUFA treatment) or OO (for the MUFA treatment). For the PUFA treatment, $50 \%$ of total fat content was derived from PUFA. For the MUFA treatment, $59 \%$ of total fat content was derived from MUFA, with differences reflecting the FA composition of those oils. The total energy intake was prescribed to equal each participant's total estimated daily energy needs (determined from RMR at 'Baseline visit' section).

Post-diet testing visit (visit 2). The day after the 5th day of the feeding period, participants reported to the laboratory under fasted $(8-12 \mathrm{~h})$ and unexercised $(12 \mathrm{~h})$ conditions. All procedures that took place during visit 1 were repeated. This included consumption of the same HF meals, and metabolic and anthropometric measurements.

The duration of the lead-in diet through visit 2 represented trial 1. The participant then underwent 2-4 weeks of washout, during which time they returned to their normal dietary patterns. After the washout period, the participants underwent trial 2 (including lead-in diet, visit 1 test-day, a 5-d high PUFA or MUFA dietary intervention and visit 2 test-day). The only difference between the trials was the foods for each 5-d trial and corresponding HF meal challenges during the testing visits (MUFA $v$. PUFA).

Table 2. Nutrient content for each high-fat (HF) test meal

\begin{tabular}{lcc}
\hline Composition & PUFA-rich HF meal & MUFA-rich HF meal \\
\hline Percentage of total energy from & & \\
Protein & 15.5 & $15 \cdot 4$ \\
Carbohydrate & 28.1 & 28.3 \\
Fat & 56.7 & $56 \cdot 3$ \\
Percentage of energy from fatty acids & \\
MUFA & 11.5 & 36.7 \\
PUFA & 31.1 & 7.4 \\
$n-6$ PUFA & 30.9 & $6 \cdot 8$ \\
$n-3$ PUFA & 0.2 & 0.6 \\
SFA & 14.2 & 12.3 \\
\hline
\end{tabular}




\section{Calculations}

Respiratory gases were used to calculate EE using the Weir equation $^{(23)}$ and macronutrient oxidation using the following equations developed by Frayn ${ }^{(25)}$ : fat $(\mathrm{g} / \mathrm{min})=\left(1.67 \times \mathrm{VO}_{2}\right.$ (litres $/ \mathrm{min}))-\left(1.67 \times \mathrm{VCO}_{2}\right.$ (litres $\left.\left./ \mathrm{min}\right)\right) ;$ and carbohydrate $(\mathrm{g} / \mathrm{min})=\left(4.56 \times \mathrm{VCO}_{2}(\right.$ litres $\left./ \mathrm{min})\right)-\left(3 \cdot 21 \times \mathrm{VO}_{2}(\right.$ litres $\left./ \mathrm{min})\right)$. $\mathrm{VO}_{2}$ and $\mathrm{VCO}_{2}$ represent $\mathrm{O}_{2}$ consumption and $\mathrm{CO}_{2}$ production, respectively, in litres/min. RER was calculated as the ratio of $\mathrm{VCO}_{2}: \mathrm{VO}_{2}$. For these calculations, the first 5 min of each 20 -min segment were discarded to allow participants to enter into a steady state. The DIT was calculated from postprandial EE subtracted by baseline EE.

In addition, the metabolic cart was calibrated against methanol burns throughout the duration of the study ${ }^{(26)}$. The percentage recoveries from each burn were used as correction factors for the corresponding metabolic data for each testing day. The average correction factors were 99.15 and $98.57 \%$ for $\mathrm{O}_{2}$ and $\mathrm{CO}_{2}$, respectively.

\section{Statistical analysis}

To determine whether our sample size was appropriate, a withinsubject, repeated-measure ANOVA was used to assess trial effects. With an effect size of 0.67 (determined from the mean postprandial change between PUFA $v$. MUFA for RER from this study), we would require a total sample size of 8 subjects, assuming $80 \%$ power $(\beta)$ and an $\alpha$ of 0.05 . The number of subjects required was determined using G*Power 3.1.9.2. SAS version 9.4 statistical package (SAS Institute Inc.) was used for all statistical analyses. To address our primary outcome of the effect of the 5-d intervention diet, change from visit 1 (before diet) to visit 2 (after diet) was calculated and a within-subjects repeated-measures ANOVA was used to detect main effects (time, treatment) and interaction effects. A secondary analysis was performed to determine differences in acute meal response at visit 1, before the intervention diet between MUFA and PUFA using a within-subject repeated-measures ANOVA. If significance was found, post hoc analyses were performed using Tukey's test. Data are presented as means with their standard errors. Statistical significance was set at $P \leq 0.05$.

\section{Results \\ Subjects}

A total of fifteen normal-weight male participants completed both feeding trials. Physical characteristics of the participants are shown in Table 3. Participants were between 18 and 45 years of age and were of normal weight based on BMI $\left(18-24.9 \mathrm{~kg} / \mathrm{m}^{2}\right)$ or DXA body fat percentage (14-24\%). All participants were also free from any known metabolic or chronic disease and were not taking any medications.

\section{Acute metabolic responses}

There were no significant differences at fasting/baseline for the pre-diet visit (visit 1) between MUFA and PUFA for any
Table 3. Participant characteristics (Mean values and standard deviations; $n$ 15)

\begin{tabular}{lrr}
\hline Characteristics & Mean & SD \\
\hline Age (years) & 21.67 & 2.58 \\
Height $(\mathrm{cm})$ & 177.74 & 6.95 \\
Weight $(\mathrm{kg})$ & 77.24 & 13.61 \\
BMl $\left(\mathrm{kg} / \mathrm{m}^{2}\right)$ & 24.27 & 2.81 \\
Body fat $(\%)$ & 16.49 & 4.85 \\
Waist circumference $(\mathrm{cm})$ & 83.61 & 9.16 \\
Hip circumference $(\mathrm{cm})$ & 98.44 & 8.54 \\
\hline
\end{tabular}

outcome variable (RER, fat and carbohydrate oxidation and EE) (Table 4). When examining the time-course meal responses, as expected, there was a significant time effect for all substrate oxidation (RER, fat and carbohydrate oxidation) and EE data (DIT) $(P<0 \cdot 001)$. There were no treatment by time interactions; however, several treatment effects were found. For RER, there was a significant treatment effect (Fig. 2(a)), with a significantly higher RER for the PUFA pre-diet visit compared with the MUFA pre-diet visit (postprandial average of 0.86 (SEM 0.01) $v$. 0.84 (sem 0.01 ),$P<0.05$, Fig. 2(b)). For fat oxidation, there also was a significant treatment effect (Fig. 3(a)), with lower fat oxidation for the PUFA pre-diet $v$. MUFA pre-diet visits (postprandial average of 1.20 (SEM 0.12) v. 1.33 (SEM 0.09) g, $P<0.05$, Fig. 3(b)). For carbohydrate oxidation, there was a significant treatment effect (Fig. 4(a)), with higher carbohydrate oxidation for the PUFA pre-diet $v$. MUFA pre-diet visit (postprandial average of 3.30 (SEM 0.17) v. 2.97 (sEm 0.18) g, $P<0.05$, Fig. 4(b)). For DIT, there was a significant treatment effect (Fig. 5(a)), with lower DIT for the PUFA pre-diet visit $v$. MUFA pre-diet visit (postprandial averages of 18.91 (SEM 0.46) v. 21.46 (sEM 1.34) kJ, $P<0 \cdot 05$, Fig. 5(b)).

\section{Metabolic responses to 5 -d high-fat diets}

There were no significant differences in fasting values before and after the diet in any outcome variable (RER, fat and carbohydrate oxidation and EE) (Table 4). When examining the change in time-course meal responses, as expected, there was a significant time effect for all substrate oxidation (RER, fat and carbohydrate oxidation) and EE data (DIT) $(P<0.001)$. There were no treatment by time interactions; however, there were several treatment effects for substrate oxidation. For change in RER, there was a significant treatment effect, with the decrease in RER from pre- to post-PUFA diet being significantly different from MUFA (postprandial average change of -0.02 (SEM 0.01) $v$. 0.00 (sem 0.01), $P<0 \cdot 05$, Fig. 6(a)).

Similar to RER, there was a significant treatment effect for change in total fat oxidation before and after the diet. The increase in the change in fat oxidation was significantly different from the change in MUFA (postprandial average change of $0 \cdot 18$ (sem 0.07) v. 0.04 (sem 0.06) g, $P<0.05$, Fig. 6(b)). For change in carbohydrate oxidation, there was also a significant treatment effect. The decrease in carbohydrate oxidation from pre- to post-PUFA diet intervention was significantly different from the change in MUFA (postprandial average change of -0.38 (SEM $0 \cdot 18) v \cdot 0 \cdot 00($ SEM $0 \cdot 17) \mathrm{g}, P<0 \cdot 05$, Fig. 6(c)). Finally, to look at 
Table 4. Fasting metabolic measurements* (Mean values with their standard errors)

\begin{tabular}{|c|c|c|c|c|c|c|c|c|}
\hline & \multicolumn{4}{|c|}{ PUFA-rich HF diet } & \multicolumn{4}{|c|}{ MUFA-rich HF diet } \\
\hline & \multicolumn{2}{|c|}{ Pre-diet } & \multicolumn{2}{|c|}{ Post-diet } & \multicolumn{2}{|c|}{ Pre-diet } & \multicolumn{2}{|c|}{ Post-diet } \\
\hline & Mean & SEM & Mean & SEM & Mean & SEM & Mean & SEM \\
\hline RER & 0.84 & 0.02 & 0.83 & 0.01 & 0.83 & 0.01 & 0.83 & 0.01 \\
\hline Fat oxidation (g/15 min) & 1.06 & 0.13 & $1 \cdot 15$ & 0.07 & $1 \cdot 14$ & 0.11 & $1 \cdot 12$ & 0.09 \\
\hline Carbohydrate oxidation ( $\mathrm{g} / 15 \mathrm{~min}$ ) & 2.65 & 0.27 & $2 \cdot 33$ & 0.19 & $2 \cdot 18$ & 0.23 & 2.32 & 0.20 \\
\hline Energy expenditure (kJ/15 min) & $80 \cdot 46$ & 3.43 & $82 \cdot 30$ & 2.93 & 78.03 & 3.43 & $80 \cdot 88$ & $2 \cdot 85$ \\
\hline
\end{tabular}

HF, high fat.

* No significant differences before and after diet or between diets.

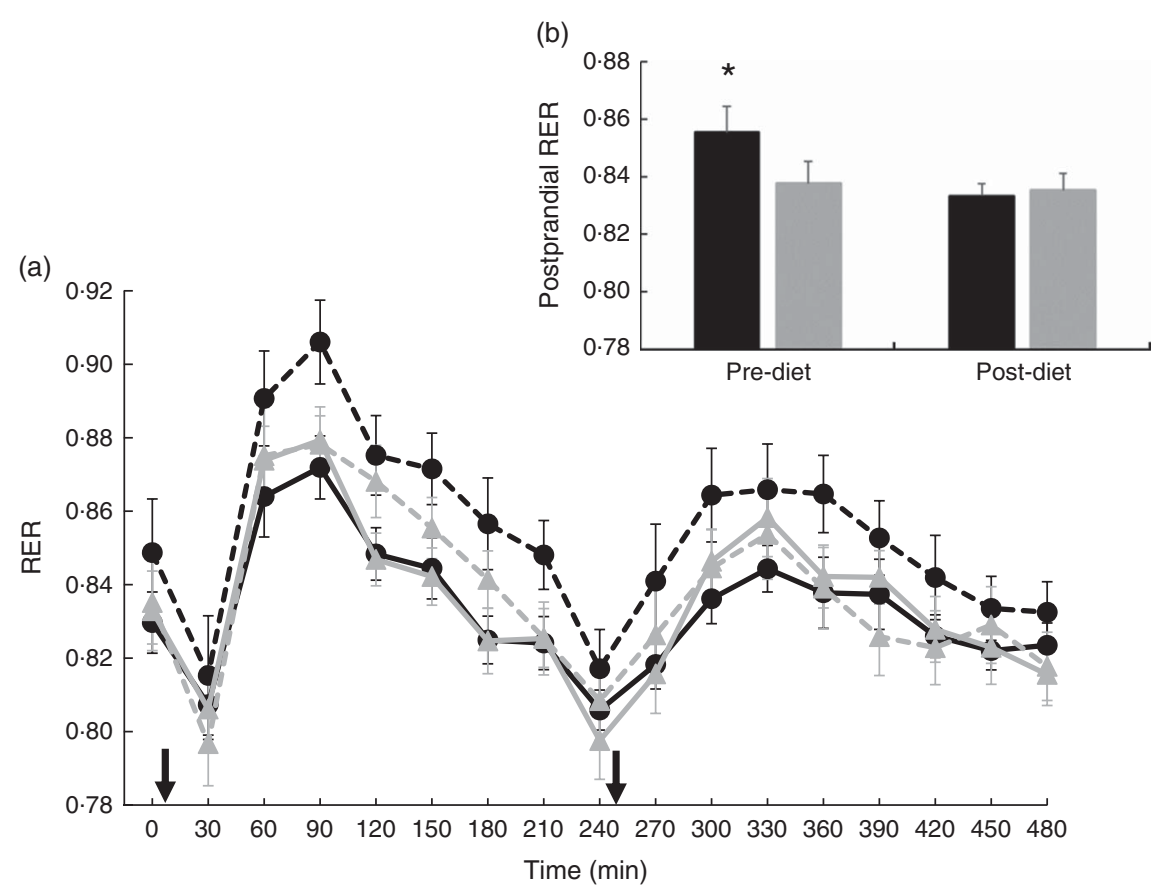

Fig. 2. (a) Fasting and postprandial RER for each of the diets, pre- and post-diet intervention. Subjects consumed the high-fat (HF) meal immediately after time 0 and time $240(\rightarrow)$. (b) Average postprandial RER for pre- and post-diet interventions. Values are means with their standard errors. a: - - - PUFA pre-diet; $-0-$, PUFA post-diet; $-A_{-}^{-}$, MUFA pre-diet; $\_$, MUFA post-diet; b: $\square$, PUFA; $\square$, MUFA. * Significantly different RER values between PUFA pre-diet and MUFA pre-diet visits $(P<0.05)$.

the meal response EE, we calculated DIT and compared the change in DIT for PUFA v. MUFA. Unlike substrate oxidation, there were no significant treatment effects for change in DIT (Fig. 6(d)).

\section{Discussion}

In this study, we found differences in the metabolic response to acute HF meal challenges compared with longer-term HF diet responses rich in PUFA or MUFA. In regard to acute meal consumption, our results indicate that intake of a HF meal rich in MUFA leads to greater fat oxidation and lower carbohydrate oxidation compared with a HF meal rich in PUFA. CSO, which was our PUFA treatment oil, is rich in $n$ - 6 FA, especially linoleic acid. A prior stable isotope study by Jones et al. ${ }^{(9)}$ also found that a meal high in oleic acid (MUFA) resulted in higher fat oxidation than a meal high in linoleic acid ( $n-6$ PUFA). Further, Delany et al. ${ }^{(10)}$ also showed that with labelled isotopes a meal high in oleic acid (MUFA) resulted in higher fat oxidation than a meal high in linoleic acid (PUFA). More recent studies have not detected a metabolic difference between acute PUFA- $v$. MUFArich meals using indirect calorimetry, and this may be owing to PUFA-rich meals having mixed contents of $n-3$ and $n-6$ PUFA $^{(12,13)}$. Delany et al. ${ }^{(10)}$ also demonstrated that a meal high in $\alpha$-linolenic acid ( $n$-3 PUFA) resulted in the greatest amount of fat oxidation compared with both oleic and linoleic acid, indicating that the type of PUFA may be important. Our study used an oil that was specifically high in linoleic acid with little $\alpha$ linolenic acid, which could have affected our significant findings compared with the null findings between MUFA and PUFA of other studies that used HF meals with significant amounts of $n-3 \operatorname{PUFA}^{(12,13)}$. 
(a)

(b)

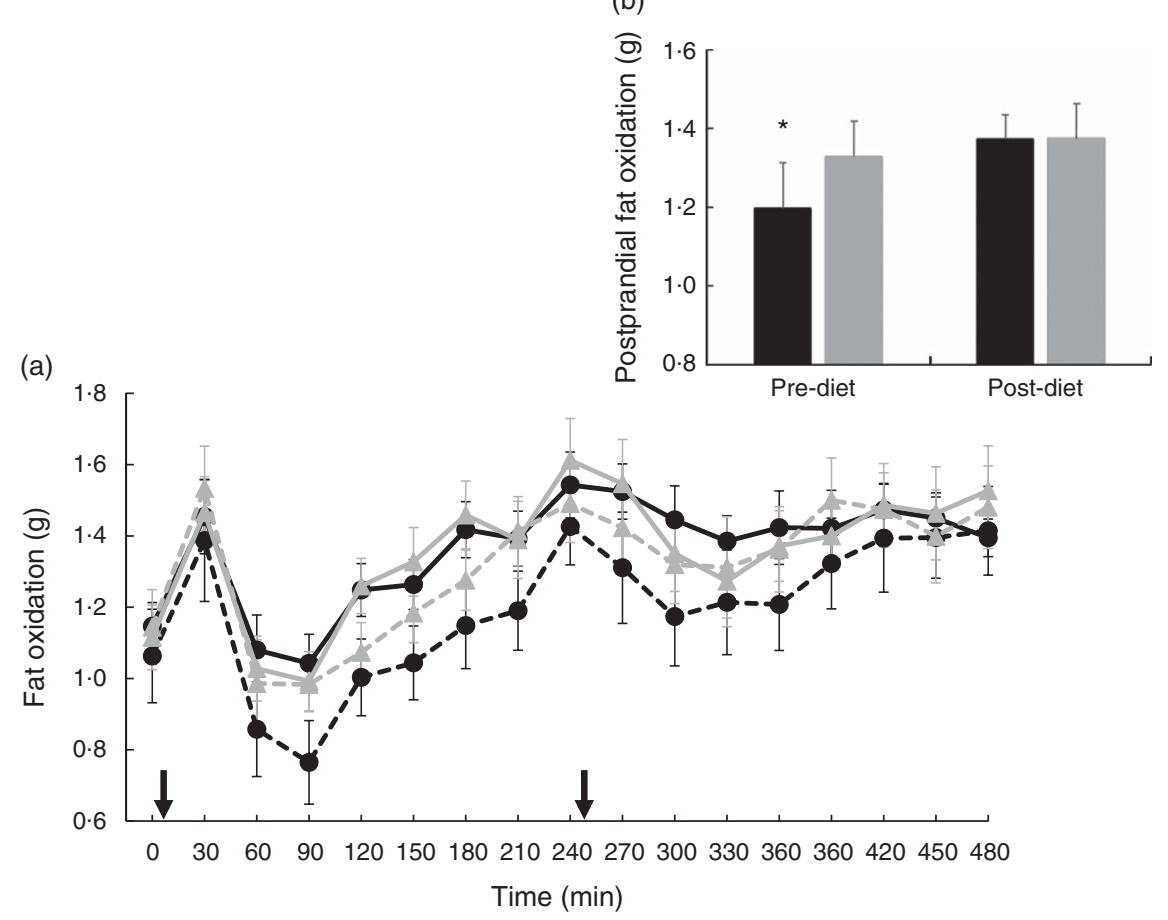

Fig. 3. (a) Fasting and postprandial fat oxidation for each of the diets, pre- and post-diet intervention. Subjects consumed the high-fat (HF) meal immediately after time 0 and time $240(\rightarrow)$. (b) Average postprandial fat oxidation for pre- and post-diet interventions. Values are means with their standard errors. a: - $-\mathbf{-}$, PUFA prediet; $-\longrightarrow$, PUFA post-diet; $-\triangle-$, MUFA pre-diet; $\_$, MUFA post-diet; b: $\square$, PUFA; $\square$, MUFA. * Significantly different fat oxidation values between PUFA pre-diet and MUFA pre-diet visits $(P<0.05)$.

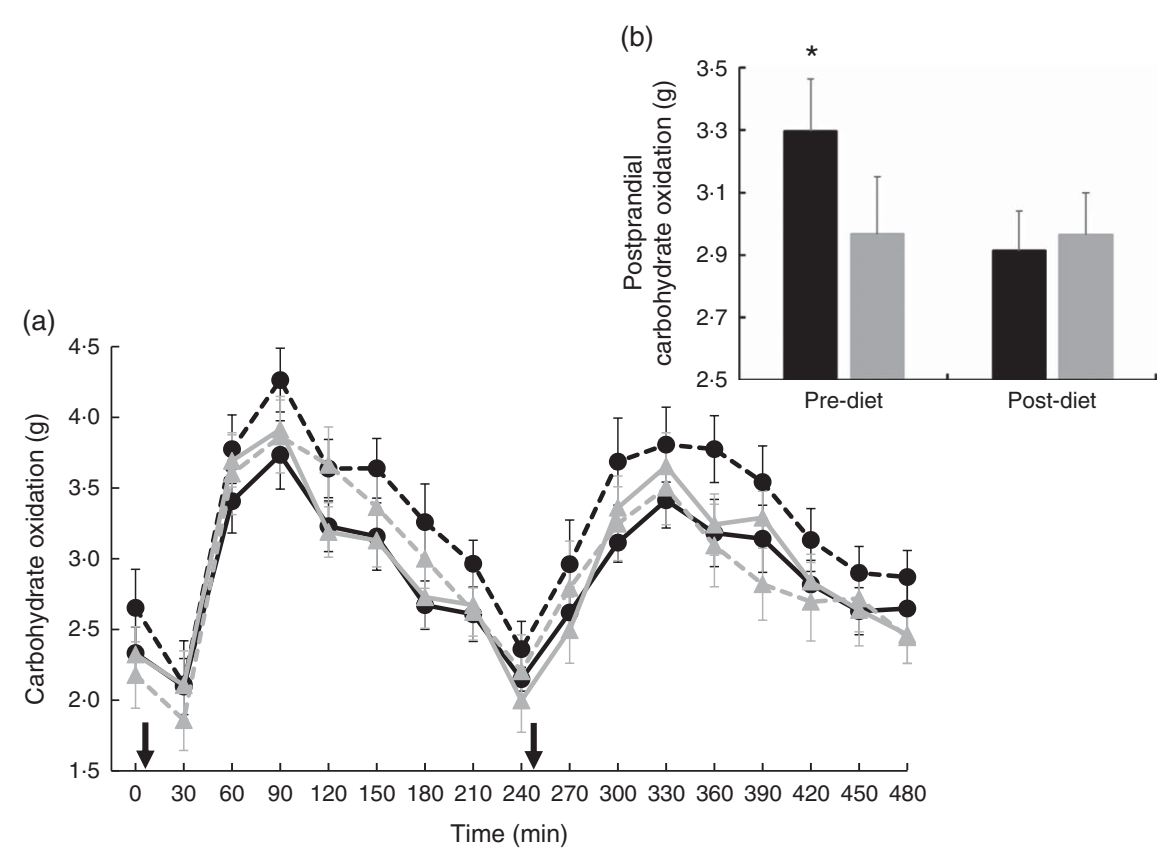

Fig. 4. (a) Fasting and postprandial carbohydrate oxidation for each of the diets, pre- and post-diet intervention. Subjects consumed the high-fat (HF) meal immediately after time 0 and time $240(\rightarrow)$. (b) Average postprandial carbohydrate oxidation for pre- and post-diet interventions. Values are means with their standard errors. a: $-\bullet-$, PUFA pre-diet; $-\longrightarrow$, PUFA post-diet; $-\perp-$, MUFA pre-diet; $\_-$, MUFA post-diet; b: $\square$, PUFA; $\square$, MUFA. * Significantly different carbohydrate oxidation values between PUFA pre-diet and MUFA pre-diet visits $(P<0.05)$.

The mechanisms behind greater fat oxidation acutely following a MUFA-rich, HF meal compared with a PUFA-rich, HF meal are largely unknown. McCloy et $a$. $^{(27)}$ demonstrated that, acutely, linoleate is preferentially esterified to plasma phospholipids and cholesteryl esters compared with oleate and $\alpha$-linolenate, which would lead to preferential retention and sequestration of linoleate rather than oxidation. However, whether this decreased oxidation of linoleate is due to a greater 


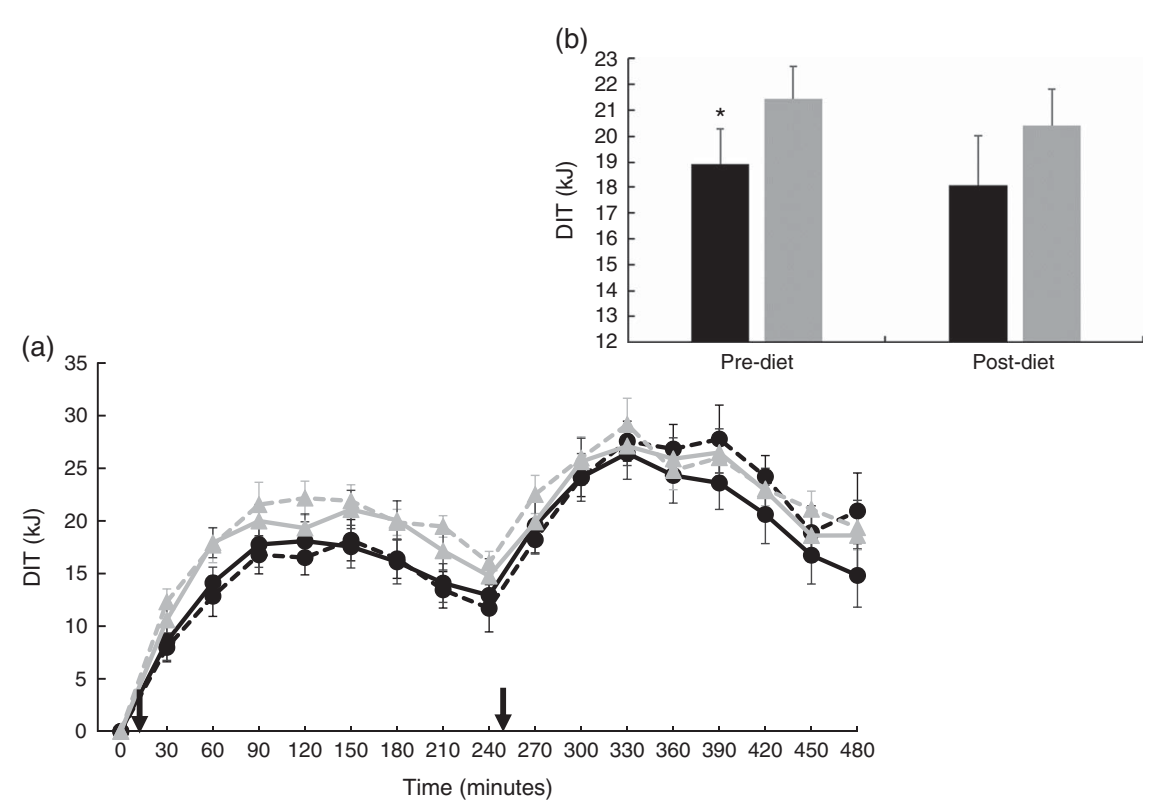

Fig. 5. (a) Diet-induced thermogenesis (DIT) for each of the diets, pre- and post-diet intervention. Subjects consumed the high-fat (HF) meal immediately after time 0 and time $240(\rightarrow)$. (b) Average DIT for pre- and post-diet interventions. Values are means with their standard errors. a: $-\infty-$, PUFA pre-diet; $\rightarrow-$ PUFA post-diet; $-\rightarrow-$, MUFA pre-diet; - , MUFA post-diet; b: $\square$, PUFA; $\square$, MUFA. * Significantly different DIT values between PUFA pre-diet and MUFA pre-diet visits.

(a)



(b)

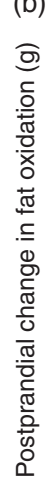

(c)

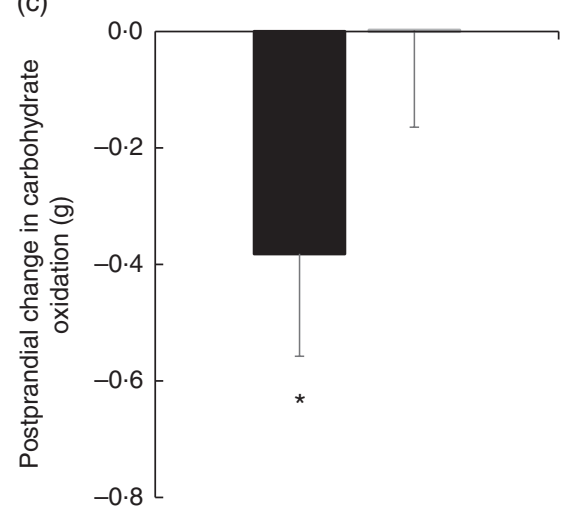

(d)

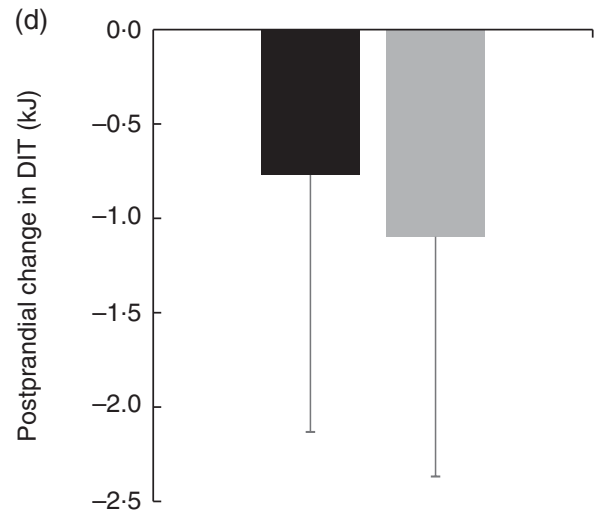

Fig. 6. (a) Pre- to post-diet change in RER for each diet. (b) Pre- to post-diet change in fat oxidation for each diet. (c) Pre- to post-diet change in carbohydrate oxidation for each diet. (d) Pre- to post-diet change in diet-induced thermogenesis (DIT) for each diet. Values are means with their standard errors. $\square$, PUFA; $\square$, MUFA. * Significantly different between PUFA and MUFA diets $(P<0.05)$. 
preference for glycerolipid synthesis, a reduced preference for $\beta$-oxidation or perhaps a combination thereof is not known. Hodson $e \mathrm{al}^{(28)}$ also demonstrated preferential incorporation of linoleate into plasma phospholipids and cholesteryl esters compared with oleate following a HF meal. This again could imply that more oleate is available for oxidation, and may explain our results of greater fat oxidation following a single MUFA-rich HF meal compared with a PUFA-rich HF meal.

Our findings also suggest that a more chronic or longer-term effect on metabolism from either PUFA or MUFA consumption is different from that of the acute meal effect. After the 5-d HF diet period, significant changes in substrate oxidation only occurred following the HF PUFA-rich diet, which led to substrate oxidation being similar between our PUFA and MUFA groups post-diet. This finding is important because it shows the adaptability of the PUFA-rich, HF diet after just $5 \mathrm{~d}$. Acutely, MUFA may have more metabolically favourable outcomes with higher fat oxidation than PUFA; however, after $5 \mathrm{~d}$ of either a HF MUFA- or PUFA-rich diet, both result in favourable metabolic measures (higher fat oxidation and lower carbohydrate oxidation). This adaptability of our PUFA-rich diet may be due to a limited capacity to sequester linoleic acid into phospholipids and thus be diverted to oxidation once this pool is full, which would be detectable with a longer-term diet ${ }^{(28)}$. Acutely, the partitioning of linoleic acid may result in preferential incorporation of these dietary fats into phospholipids, hence lower oxidation after a single meal ${ }^{(27,28)}$. However, longer-term consumption of a diet rich in linoleic acid, such as our PUFA diet, is able to reveal changes and adaptations over a few days. PUFA are also potent regulators of lipogenic gene expression, and diets rich in linoleic acid result in down-regulation of genes encoding enzymes of lipid synthesis ${ }^{(29)}$, thus affirming our findings of increased fat oxidation after a 5-d, PUFA-rich diet. Finally, the CSO used to enrich the PUFA diet in this study may contain a unique component that could be contributing to the increase in oxidation seen after our 5-d diet. CSO contains cyclopropenoid FA, specifically dihydrosterculic acid (DHSA $)^{(30)}$. DHSA may play an important role in reducing stearoyl-CoA desaturase-1 $\operatorname{activity}^{(30,31)}$, therefore reducing overall TAG formation and channelling free FA into $\beta$-oxidation $^{(32)}$. This illustrates a unique component that may be present in CSO. Taken together, the significant increase in fat oxidation from pre- to post-PUFA-rich HF diet may have been owing to the FA content, as well as a unique component in the CSO used in the diet.

Other longer-term studies have investigated the metabolic effects of PUFA $v$. SFA ${ }^{(6,20,21)}$ and MUFA $v$. SFA ${ }^{(17-19)}$. In addition, our laboratory previously showed that a 7-d, PUFA-rich diet resulted in higher fat oxidation following a SFA-rich meal compared with a control diet (containing a higher percentage of fat from MUFA) ${ }^{(22)}$. In contrast, we did not see differences between our MUFA- and PUFA-rich diets after $5 \mathrm{~d}$, leading us to reject our hypothesis that a PUFA-rich HF diet would lead to greater fat oxidation than a MUFA-rich HF diet. This could be owing to the relatively $\mathrm{HF}$ content of our diets, which could mask the FA effect that was detectable in our previous study, with a lower percentage of energy in the diet being fat ${ }^{(22)}$. Our previous PUFA-rich, 7-d diet intervention also had a higher percentage of PUFA coming from $n-3$ fats, which may have also contributed to differences between the two study outcomes ${ }^{(22)}$.

Our acute results for DIT showed that a HF, MUFA-rich meal resulted in greater DIT compared with a HF, PUFA-rich meal. In agreement with these results, Jones et al. ${ }^{(11)}$ found that a HF meal enriched in MUFA induced greater DIT compared with a HF meal enriched in PUFA. However, in contrast to our findings, Clevenger et al. ${ }^{(13)}$ found that a HF meal rich in PUFA increases postprandial thermogenesis compared with a MUFArich meal in females. Potential metabolic differences between males and females may contribute to the contradicting results of the current study. Other previous studies have not detected differences in DIT between MUFA and PUFA ${ }^{(12,33)}$. It is also important to note that while the change in DIT was not different between MUFA and PUFA for the 5-d diet period, DIT was similar between PUFA and MUFA after the 5-d diet intervention period.

Limitations of the current study include self-reported meal compliance during the study, which could affect the outcomes measured. Our participants were free-living, making it possible for them to consume other foods and beverages throughout the day. However, participants came into the laboratory every morning to consume breakfast and were reminded each day to only eat and drink the food that was provided to them. Another limitation of the study is that we used oils high in either PUFA or MUFA to enrich each diet type, and such oils may contain other beneficial nutrients or components in them that could be attributable to the metabolic differences. For example, OO contains polyphenols that may inhibit lipogenesis, therefore possibly contributing to its effect in greater fat oxidation ${ }^{(34)}$. However, because the study design was matched except for differences in oils used, we can narrow differences down to the specific food source (CSO or OO). Future studies are needed to determine whether these differences are specific to the FA in the oils used, owing to other nutrients within the oils, or a combination of a synergistic effect of the nutrients and types of FA in the oil as a whole. Another limitation is that the percentage of fat from our FA of interest (MUFA for OO, PUFA for CSO) differed because of the composition of the oils $(67 \cdot 1 \%$ MUFA in OO, $57 \cdot 3 \%$ PUFA in CSO). However, one of the strengths of the current study is that we used a whole foods approach, mimicking reallife eating patterns. As we do not eat FA, matching the percentage of total energy from a specific FA group is not always feasible. Our study also contained diets that were above the upper limits of dietary recommendations for total fat and PUFA content, which was by design to establish proof of principle. This may, however, limit clinical application of the diets used, and future studies are needed to explore the metabolic responses to lower fat diets. Last, the study sample included apparently healthy men with normal body fat percentages, and thus these results may not be extrapolated to other populations.

In conclusion, the present findings indicate that an acute $\mathrm{HF}$ meal rich in MUFA from OO results in greater acute total fat oxidation and lower total carbohydrate oxidation and RER compared with a HF meal rich in PUFA from CSO. However, consumption of a 5-d HF diet rich in PUFA leads to increases in total fat oxidation and decreases in total carbohydrate oxidation and RER, making substrate oxidation after 5-d HF diets rich in 
these different fats equal. Because greater fat oxidation has been associated with lower body fat mass, rates of fat oxidation can have implications for weight management. Our results indicate that after a 5-d period both sources of unsaturated FA may have a similar metabolic impact on weight management as fat oxidation rates were similar between the diets. Finally, DIT followed the same trend as seen with substrate oxidation, being higher after the acute MUFA-rich meal compared with PUFA. Although the change over the 5-d HF diet intervention was not significantly different between treatments, DIT was similar between the treatments after adaptation occurred with the PUFA-rich diet. Future studies are needed to investigate the mechanisms behind differences seen acutely between PUFA and MUFA, and whether further differences would be seen with chronic consumption of these two diets.

\section{Acknowledgements}

This research project was funded by Cotton Incorporated. Research was also supported by the AU/UGA Medical Partnership and UGA Clinical and Translational Research Unit.

J. A. C. and C. M. P. contributed to the conception and design of the project, as well as interpretation and providing important intellectual content and review of the preparation of the manuscript. R. V., M. J., M. K. M. and K. R. P. contributed to data acquisition. M. K. M. and K. R. P. contributed to analysis and K. R. P. also contributed to interpretation, manuscript preparation and writing of the final manuscript.

None of the authors has any conflicts of interest to declare.

\section{References}

1. Dietary Guidelines Advisory Committee (2015) Scientific Report of the 2015 Dietary Guidelines Advisory Committee. Washington, DC: USDA and US Department of Health and Human Services.

2. Swinburn BA, Caterson I, Seidell JC, et al. (2004) Diet, nutrition and the prevention of excess weight gain and obesity. Public Health Nutr 7, 123-146.

3. Krishnan S \& Cooper JA (2014) Effect of dietary fatty acid composition on substrate utilization and body weight maintenance in humans. Eur J Nutr 53, 691-710.

4. Raatz SK, Conrad Z, Johnson LK, et al. (2017) Relationship of the reported intakes of fat and fatty acids to body weight in US adults. Nutrients $\mathbf{9}, 438$.

5. Westerterp KR, Smeets A, Lejeune MP, et al. (2008) Dietary fat oxidation as a function of body fat. Am J Clin Nutr 87, 132-135.

6. Jones PJ \& Schoeller DA (1988) Polyunsaturated: saturated ratio of diet fat influences energy substrate utilization in the human. Metabolism 37, 145-151.

7. Leyton J, Drury P \& Crawford M (1987) Differential oxidation of saturated and unsaturated fatty acids in vivo in the rat. BrJ Nutr 57, 383-393.

8. Piers LS, Walker KZ, Stoney RM, et al. (2002) The influence of the type of dietary fat on postprandial fat oxidation rates: monounsaturated (olive oil) vs saturated fat (cream). Int J Obes Relat Metab Disord 26, 814-821.

9. Jones P, Pencharz P \& Clandinin M (1985) Absorption of 13Clabeled stearic, oleic, and linoleic acids in humans: application to breath tests. J Lab Clin Med 105, 647-652.
10. DeLany JP, Windhauser MM, Champagne CM, et al. (2000) Differential oxidation of individual dietary fatty acids in humans. Am J Clin Nutr 72, 905-911.

11. Jones PJ, Jew S \& AbuMweis S (2008) The effect of dietary oleic, linoleic, and linolenic acids on fat oxidation and energy expenditure in healthy men. Metabolism 57, 1198-1203.

12. Casas-Agustench P, Lopez-Uriarte P, Bullo M, et al. (2009) Acute effects of three high-fat meals with different fat saturations on energy expenditure, substrate oxidation and satiety. Clin Nutr 28, 39-45.

13. Clevenger HC, Kozimor AL, Paton CM, et al. (2014) Acute effect of dietary fatty acid composition on postprandial metabolism in women. Exp Physiol 99, 1182-1190.

14. Bray GA (1990) Obesity - a state of reduced sympathetic activity and normal or high adrenal activity (the autonomic and adrenal hypothesis revisited). Int J Obes 14, Suppl. 3, 77-91; discussion 91-72.

15. Tappy L (1996) Thermic effect of food and sympathetic nervous system activity in humans. Reprod Nutr Dev 36, 391-397.

16. Soares MJ, Cummings SJ, Mamo JC, et al. (2004) The acute effects of olive oil $\mathrm{v}$. cream on postprandial thermogenesis and substrate oxidation in postmenopausal women. Br J Nutr 91, 245-252.

17. Kien CL, Bunn JY \& Ugrasbul F (2005) Increasing dietary palmitic acid decreases fat oxidation and daily energy expenditure. Am J Clin Nutr 82, 320-326.

18. Kien CL \& Bunn JY (2007) Effects of palmitate and oleate on the respiratory quotient during acute feeding. Obesity (Silver Spring) 15, 1640-1642.

19. Piers LS, Walker KZ, Stoney RM, et al. (2003) Substitution of saturated with monounsaturated fat in a 4-week diet affects body weight and composition of overweight and obese men. Br J Nutr 90, 717-727.

20. van Marken Lichtenbelt WD, Mensink RP \& Westerterp KR (1997) The effect of fat composition of the diet on energy metabolism. Z Ernabrungswiss 36, 303-305.

21. Jones PJ, Ridgen JE, Phang PT, et al. (1992) Influence of dietary fat polyunsaturated to saturated ratio on energy substrate utilization in obesity. Metabolism 41, 396-401.

22. Stevenson JL, Miller MK, Skillman HE, et al. (2016) A PUFArich diet improves fat oxidation following saturated fatrich meal. Eur J Nutr 56, 1845-1857.

23. Weir JB (1949) New methods for calculating metabolic rate with special reference to protein metabolism. J Physiol 109, 1-9.

24. Trumbo P, Schlicker S, Yates AA, et al. (2002) Dietary reference intakes for energy, carbohydrate, fiber, fat, fatty acids, cholesterol, protein and amino acids. J Am Diet Assoc 102, $1621-1630$.

25. Frayn KN (1983) Calculation of substrate oxidation rates in vivo from gaseous exchange. J Appl Physiol Respir Environ Exerc Physiol 55, 628-634.

26. Schoffelen PF, Westerterp KR, Saris WH, et al. (1997) A dualrespiration chamber system with automated calibration. J Appl Physiol 83, 2064-2072.

27. McCloy U, Ryan MA, Pencharz PB, et al. (2004) A comparison of the metabolism of eighteen-carbon 13C-unsaturated fatty acids in healthy women. $J$ Lipid Res $\mathbf{4 5}$, 474-485.

28. Hodson L, McQuaid SE, Karpe F, et al. (2009) Differences in partitioning of meal fatty acids into blood lipid fractions: a comparison of linoleate, oleate, and palmitate. Am J Physiol Endocrinol Metab 296, E64-E71.

29. Sampath H \& Ntambi JM (2005) Polyunsaturated fatty acid regulation of genes of lipid metabolism. Annu Rev Nutr 25, 317-340.

30. Paton CM, Vaughan RA, Selen Alpergin ES, et al. (2017) Dihydrosterculic acid from cottonseed oil suppresses desaturase activity and improves liver metabolomic profiles of high-fat-fed mice. Nutr Res 45, 52-62. 
31. Gomez FE, Bauman DE, Ntambi JM, et al. (2003) Effects of sterculic acid on stearoyl-CoA desaturase in differentiating 3T3-L1 adipocytes. Biochem Biophys Res Commun 300, 316-326.

32. Paton CM \& Ntambi JM (2009) Biochemical and physiological function of stearoyl-CoA desaturase. Am J Physiol Endocrinol Metab 297, E28-E37.
33. Flint A, Helt B, Raben A, et al. (2003) Effects of different dietary fat types on postprandial appetite and energy expenditure. Obes Res 11, 1449-1455.

34. Rigacci S \& Stefani M (2016) Nutraceutical properties of olive oil polyphenols. an itinerary from cultured cells through animal models to humans. Int J Mol Sci 17, E843. 